\title{
The future cost of cancer in South Africa: An interdisciplinary cost management strategy
}

In the guest editorial entitled 'The future cost of cancer in South Africa: An interdisciplinary cost management strategy', which appeared in the October 2016 SAMJ (106(10):949-950), author V Sharma's affiliation should have read: 'Head, Department of Radiation Oncology, Charlotte Maxeke Johannesburg Academic Hospital and Faculty of Health Sciences, University of the Witwatersrand, Johannesburg, South Africa. The online version of the article (http://dx.doi.org/10.7196/SAMJ.2016.v106i10.11375) was corrected on 25 November 2016.

S Afr Med J 2016;106(12):1270. DOI:10.7196/SAMJ.2016.v106i12.12182 